\title{
Rapid tip-directed movement of Golgi equivalents in growing Aspergillus nidulans hyphae suggests a mechanism for delivery of growth-related materials
}

Correspondence

Susan G. W. Kaminskyj

Susan.Kaminskyj@usask.ca

Received 7 November 2007

Revised 19 February 2008

Accepted 20 February 2008

\author{
Michelle A. Hubbard and Susan G. W. Kaminskyj
}

Department of Biology, University of Saskatchewan, 112 Science Place, Saskatoon, SK S7N 5E2, Canada

\begin{abstract}
Golgi equivalents (GEs) process materials in the fungal secretory pathway. Despite the importance of localized secretion in fungal tip growth, GE behaviour in living hyphae has not been documented. The distribution was monitored of an Aspergillus nidulans putative GE-associated protein, CopA, tagged with GFP (CopA-GFP). This co-localized with a Golgi body/GE marker established in other systems, $\alpha$-2,6-sialyltransferase, tagged with red fluorescent protein (ST-RFP). CopA-GFP and ST-RFP distributions responded similarly to brefeldin A, which impairs Golgi/GE trafficking. We used a CopA-GFP, hypA1 strain to study GE distribution and behaviour in growing $A$. nidulans hyphae. This strain has a wild-type phenotype at $28{ }^{\circ} \mathrm{C}$, can be manipulated by changing growth temperature or by use of cytoskeleton inhibitors, and its GE behaviour is consistent with that in a wild-type-morphology strain. A. nidulans GEs were more abundant at hyphal tips than subapically, and showed saltatory motility in all directions. Anterograde GE movements predominated. These were positively correlated with, but at least 10-fold faster than, hyphal growth rate, under all growth and experimental conditions investigated. The actin inhibitor latrunculin B reduced both anterograde GE movement and hyphal growth rate, whereas the microtubule (MT) depolymerizer benomyl increased anterograde GE movement and decreased hyphal growth rate. The MT stabilizer taxol increased $A$. nidulans GE movement but not hyphal growth rate. A. nidulans GE motility appears to have a complex dependence on both actin and MTs. We present a model for apical delivery of growth materials in which A. nidulans GEs play a role in long-distance transport.
\end{abstract}

\section{INTRODUCTION}

Fungal tip growth uses the coordinated activities of the endomembrane and cytoskeletal systems to target growth materials to the hyphal apex (Bartnicki-Garcia, 2002; Heath, 1990, 1995). Golgi bodies process and sort materials (Farquhar \& Palade, 1981, 1998; Matheson et al., 2006; Mogelsvang \& Howell, 2006), including those destined for secretion. Fungal Golgi equivalents (GEs) differ morphologically from Golgi bodies in animals and plants but perform similar functions (Beckett et al., 1974; Bentivoglio \& Mazzarello, 1998; Cole et al., 2000), making them central players in the tip growth process. As in most fungi, Aspergillus nidulans GEs imaged with transmission electron microscopy (TEM) have single pleiomorphic cisternae (Beckett et al., 1974; Kaminskyj \& Boire, 2004; Kurtz et al., 1994).

Abbreviations: BFA, brefeldin A; CaMV, cauliflower mosaic virus; GE, Golgi equivalent; MT, microtubule; RFP, red fluorescent protein; ST, $\alpha$ 2,6-sialyltransferase; TEM, transmission electron microscopy.

A supplementary figure (video clip) showing Golgi equivalent motility in Aspergillus nidulans strain $A A B 1$ is available with the online version of this paper.
The distribution of organelles (Bartnicki-Garcia, 2002) and proteins (McGoldrick et al., 1995; Sharpless \& Harris, 2002) can be used to infer their function. Evidence from $A$. nidulans (Breakspear et al., 2007), Aspergillus oryzae (Akao et al., 2006), Candida albicans (Rida et al., 2006) and Saccharomyces cerevisiae (Matsuura-Tokita et al., 2006) suggests that GE distribution in fungi is related to tip growth.

The behaviour of organelles in living A. nidulans hyphae has been revolutionized by fluorescent protein tagging (e.g. Suelmann \& Fischer, 2000a, b). Such studies depend on verified localizations, using comparisons with independent localization methods. In $A$. nidulans, $\operatorname{sod}{ }^{V I} C$ (stabilization of disomy in chromosome VI) has been shown to encode a protein with high similarity to Saccharomyces cerevisiae aCOPI (Whittaker et al., 1999). The COPI coat is required for one type of GE-derived vesicle formation (Bentivoglio \& Mazzarello, 1998; Boevink et al., 1998; Hawes \& SatiatJeunemaitre, 2005). Breakspear et al. (2007), who renamed sod ${ }^{V I} C$ as $\operatorname{cop} A$, showed that CopA-GFP localized to punctate arrays in A. nidulans hyphae. These arrays were adversely affected by brefeldin A (BFA) treatment, suggesting that they were A. nidulans GEs. Rat $\alpha$-2,6-sialyltransferase (ST) 
contains a transmembrane domain that has been shown to be important for Golgi body retention in animals (Munro, 1991) and plants (Wee et al., 1998), and for GE localization in yeast (Schwientek et al., 1995), suggesting that it is a reliable marker with which to confirm the localization of CopA-GFP.

A. Breakspear and S. S. Assinder (University of Bangor, UK) provided us with a CopA-GFP strain in a hypA1 temperature-sensitive background. The hypA gene encodes an orthologue of Saccharomyces cerevisiae Trs120p, a regulatory subunit in the COPII secretory pathway implicated in fungal GE transit (Shi et al., 2004). When grown at $28{ }^{\circ} \mathrm{C}$, hypA 1 strains have a wild-type phenotype (Kaminskyj \& Hamer, 1998; Kaminskyj \& Boire, 2004; Shi et al., 2004). When grown at $42{ }^{\circ} \mathrm{C}$, hypA1 strains have reduced cell polarity, swollen GEs and thick cell walls, but these restrictive-phenotype cells establish wild-type-morphology hyphal branches within an hour of being shifted to $28^{\circ} \mathrm{C}$ (Kaminskyj \& Hamer, 1998; Kaminskyj \& Boire, 2004; Shi et al., 2004).

Here, we confirm the localization of CopA-GFP at GEs in growing $A$. nidulans hyphae by co-localization with the ST transmembrane domain tagged with red fluorescent protein (RFP). A. nidulans GEs showed saltatory motility, with movements being predominantly anterograde. We explored the relationship between the distribution and movement of $A$. nidulans GEs with respect to cell polarity and tip growth using the hypA1 temperature-sensitive morphogenesis allele and cytoskeleton-targeting drugs. Our results suggest that long-distance delivery of growthrelated materials to the hyphal tip may employ anterograde GE movements.

\section{METHODS}

A. nidulans strains and growth conditions. The biological materials used in this study are listed in Table 1. Media and culture methods are described in Hubbard \& Kaminskyj (2007) and
Kaminskyj (2001). Unless stated otherwise, all cells for all experiments were grown at $28{ }^{\circ} \mathrm{C}$, and had a wild-type phenotype (Kaminskyj \& Hamer, 1998). Most experiments used strain AAB1 with GFP under the control of the alcA promoter (Felenbok, 1991). GFP expression was induced by growth on complete medium $(\mathrm{CM})$ containing $1 \%$ $(\mathrm{v} / \mathrm{v})$ glycerol or $1 \%$ ethanol as the sole carbohydrate source, or on Difco nutrient agar supplemented with $0.5 \%$ threonine. Anhydrous ethanol or solid threonine was added to cool, autoclaved media.

For microscopy, freshly harvested spores were inoculated onto sterile dialysis tubing overlying solid medium, and grown overnight at $28{ }^{\circ} \mathrm{C}$ (Hubbard \& Kaminskyj, 2007). For drug-treated cells, the dialysis tubing and overlying hyphae were mounted in a microscope slide chamber in $\sim 100 \mu \mathrm{l}$ liquid medium that contained solvents and cytoskeleton-selective inhibitors as required, and allowed to recover for $30 \mathrm{~min}$ before observation, as described in Hubbard \& Kaminskyj (2007). Mounting induced transient hyphal tip swelling, which made a convenient marker for identifying vigorously growing cells. Observations were terminated by $120 \mathrm{~min}$.

Some hyphae were treated with BFA. In this case, hyphae were mounted in the slide chamber in CM-ethanol or nutrient broththreonine and given $30 \mathrm{~min}$ for recovery, then medium plus BFA was added by rinsing through the slide chamber. Observations began as soon as possible following addition of BFA and were terminated after $60 \mathrm{~min}$.

Inhibitors. Benomyl, paclitaxel (trade name, Taxol) and anhydrous DMSO were from Sigma. Latrunculin B (hereafter, latrunculin) was from Molecular Probes. All other chemicals were from VWR. Inhibitors were diluted from stock solutions with room-temperature liquid medium immediately before use.

Benomyl was stored at $4{ }^{\circ} \mathrm{C}$ as a $10 \mathrm{mg} \mathrm{ml}^{-1}$ stock in $100 \%$ ethanol, and used at $1 \mu \mathrm{g} \mathrm{ml}^{-1}$ in $0.01 \%$ ethanol. Latrunculin was stored as a $25 \mathrm{mg} \mathrm{ml}^{-1}$ stock in $100 \%$ ethanol at $-20{ }^{\circ} \mathrm{C}$, and used at $5 \mu \mathrm{g} \mathrm{ml}^{-1}$ in $0.2 \%$ ethanol. Taxol was stored at $-20{ }^{\circ} \mathrm{C}$ as a $2 \mathrm{mM}$ stock in $100 \%$ DMSO and used at $50 \mu \mathrm{M}$ in $0.25 \%$ DMSO. DMSO was purchased as $1 \mathrm{ml}$ ampoules of dry solvent, and the stock was stored over desiccant. BFA was stored at $-20{ }^{\circ} \mathrm{C}$ as a $5 \mathrm{mg} \mathrm{ml}^{-1}$ stock in $100 \%$ methanol, and used at $10 \mu \mathrm{g} \mathrm{ml}^{-1}$ in $0.5 \%$ methanol. The inhibitor concentrations were similar to or lower than those typically used (benomyl, BFA and latrunculin), or were the lowest for which a response was detected (taxol) as described in Hubbard \& Kaminskyj (2007).

Confocal microscopy. A. nidulans hyphae were imaged with a Zeiss META 510 laser scanning confocal microscope with a Plan

Table 1. Strains and plasmids used in this study

\begin{tabular}{|c|c|}
\hline Strain or plasmid & Relevant characteristics \\
\hline \multicolumn{2}{|l|}{ A. nidulans strains } \\
\hline $\mathrm{AAB}^{*}$ & AlcA-CopA-GFP; hypA1, pabaA6; veA1 \\
\hline $\mathrm{AMH} 1 \dagger$ & AlcA-CopA-GFP, paba A6, yA2; pyrG89; veA1 \\
\hline $\mathrm{AMH} 2 \dagger$ & AlcA-CopA-GFP; CaMV 35S-RFP-4,6-ST; paba A6, yA2; pyrG89:: N. crassa pyr4 ${ }^{+}$, veA1 \\
\hline \multicolumn{2}{|l|}{ Plasmids } \\
\hline ARp1末 & $\begin{array}{l}\text { Autonomously replicating AMA1 plasmid, amp }{ }^{\mathrm{R}} \text {, containing } N . \text { crassa } \text { pyrt }^{+} \text {as a } \\
\text { selectable marker }\end{array}$ \\
\hline ST-RFPS & CaMV 35S-RFP-ST, amp ${ }^{R}$ \\
\hline
\end{tabular}

${ }^{\star}$ A gift from Susan Assinder and Andrew Breakspear (University of Wales, Bangor, UK).

$\dagger$ This study.

\$Shi et al. (2004).

\$A gift from Chris Hawes (Oxford Brookes University, Oxford, UK) and Federica Brandizzi (University of Saskatchewan, SK). 
Apochromat $\times 63$, numerical aperture 1.2 multi-immersion objective equipped with phase-contrast optics. CopA-GFP imaging used $488 \mathrm{~nm}$ excitation, 5-10\% power from an argon multispectral laser operated at 5.9 A, with emission controlled by a BP505-530 filter. STRFP imaging used $543 \mathrm{~nm}$ excitation, $5-10 \%$ power from a $25 \mathrm{~mW}$ HeNe1 laser, with emission controlled by an LP585 filter. For each type of imaging, eight or 16 scans at $0.6-2.5 \mu$ s per pixel were used to improve signal to noise ratio. Optical sections were $1.2 \mu \mathrm{m}$ thick, and chosen to be near-median focal level, as judged by cell profile. Observations were based on single optical sections taken from timelapse series, 5-20 images collected over 60-300 s. Fluorescence images for studying GE movement and transmitted images for hyphal growth rate were collected at the same time.

Hyphae were chosen for analysis if they were located at the colony margin, had an even width profile, had a smoothly tapered tip and had grown out from the characteristic mounting-induced morphology, that is, a swollen tip or an abrupt change in growth direction. Hyphae that had not responded in this way to mounting were assumed to be non-growing and were not selected for analysis. However, whether a particular hypha was actually growing, and at what rate, was not determined until after the data were collected.

A. nidulans containing RFP-tagged ST. A 52 aa transmembrane domain from rat ST (Munro, 1991), tagged at the C terminus with monomeric red fluorescent protein (mRFP, hereafter RFP) under the control of the cauliflower mosaic virus (CaMV) $35 \mathrm{~S}$ promoter was provided by F. Brandizzi (formerly University of Saskatchewan) and C. Hawes (Oxford Brookes University). A. nidulans strain AMH1 was transformed using $4 \mu \mathrm{g}$ ST-RFP DNA plus $1 \mu \mathrm{g}$ ARp1-pyr4 DNA, which contains Neurospora crassa pyr4 ${ }^{+}$as a selectable marker, following the procedure described in Shi et al. (2004). Transformants were grown at $28{ }^{\circ} \mathrm{C}$ for $72 \mathrm{~h}$ before colonies were tested for expression of RFP and GFP fluorescence. Strain AMH2 expressed RFP on all growth media, and expressed GFP on CM-ethanol or nutrient broth-threonine. GFP and RFP expression were probed on Western blots of $\mathrm{AAB} 1$ and $\mathrm{AMH} 2$ grown under AlcA-inducing and -suppressing conditions, respectively, and using a polyclonal antiGFP that can recognize both GFP and RFP [Santa Cruz Biotechnology Inc. (SCBI) Technical Support, personal communication]. Both constructs had bands with the expected mobility, and in addition CopA-GFP had some free GFP (see figure 3-3 in Hubbard, 2007) that likely contributed to background fluorescence.

Statistical and graphical analysis. Data are expressed as the mean \pm SEM. Statistical analyses used the 2000 version of Microsoft Excel with data analysis add-ins, which generates probability values. Statistical comparisons between treatments used one-way, singlefactor ANOVA, and post-hoc comparisons used Fisher predicted least-square difference (PLSD). Numerical data are presented using the 2000 version of Microsoft Excel. Images are presented using Adobe Photoshop 7.0 with minor contrast adjustments.

\section{RESULTS}

\section{GE distribution in A. nidulans hyphae}

In living A. nidulans hyphae, both the ST-RFP and CopAGFP markers had punctate localizations consistent with that expected for GEs (Fig. 1a, b). These were roughly $0.5-$ $1 \mu \mathrm{m}$ in diameter, and co-localized extensively (Fig. 1c). The CopA-GFP pattern had a somewhat coarse cytoplasmic background, consistent with COPI-coated vesicles that cannot be resolved by confocal fluorescence. Comparing fluorescence and transmitted images (Fig. 1c, d) at least

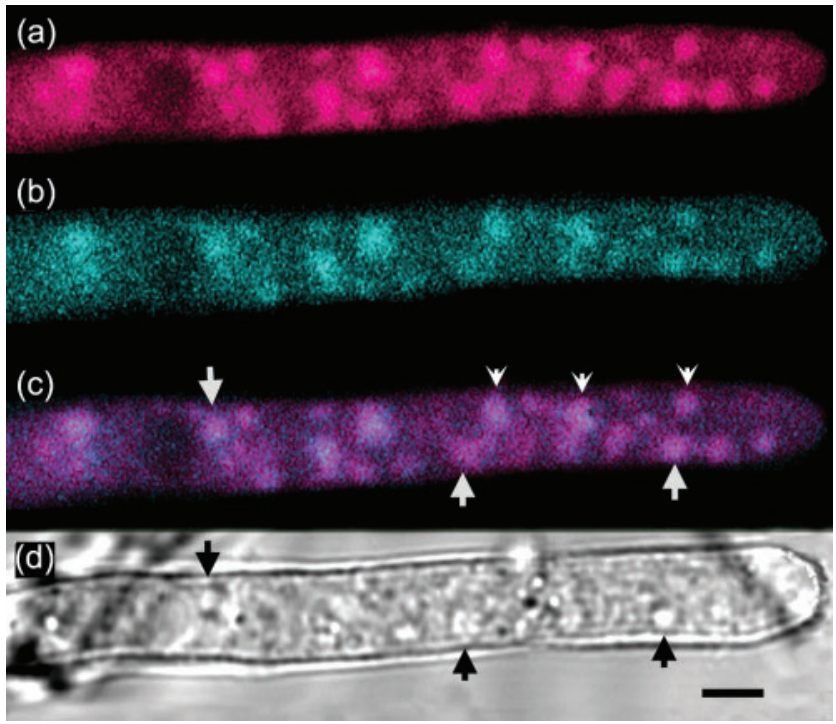

Fig. 1. Distributions of fluorescent protein-tagged GE markers in a wild-type-phenotype $A$. nidulans hypha show extensive co-localization when viewed with confocal microscopy in a near-median section. (a) Distribution of the transmembrane domain of ST, Cterminal tagged with RFP (ST-RFP), pseudo-coloured magenta. (b) Distribution of CopA, C-terminal tagged with GFP, pseudo-coloured cyan. (c) Colocalization of (a) and (b). (d) Transmitted light image. Arrows in (c) and (d) indicate punctate fluorescence in (c) that appears to co-localize with inclusions in the transmitted image in (d). Arrowheads in (c) indicate punctate fluorescence in (c) that lacks such apparent co-localization. Bar, $2 \mu \mathrm{m}$.

seven of 26 large fluorescent structures appeared to colocalize with cytoplasmic structures of a similar size that were visualized with transmitted light (arrows), although many others did not (arrowheads). Epifluorescence images viewed with confocal optics have a shallower depth of focus than their associated transmitted light images, which makes precise spatial correlations difficult. Nevertheless, this is intriguing, since GEs have not, to our knowledge, been imaged previously without staining in living fungal cells.

BFA affects endomembrane trafficking by inhibiting an early stage in COPI vesicle formation. A. nidulans hyphae treated with $10 \mu \mathrm{g} B F A m^{-1}$ had less well-defined GE patterns following 25 min of treatment (Fig. 2a-d) which is consistent with the anticipated BFA effect. After $60 \mathrm{~min}$ of BFA treatment there were preliminary indications of a reticulate network (arrows in Fig. 2e) that was entirely unlike the punctate arrays seen in untreated cells.

In wild-type-morphology A. nidulans hyphae, GEs were significantly more abundant in the apical $25 \mu \mathrm{m}$ than further back $(P<0.01$, ANOVA). This apical region had one or two GE per micrometre of hyphal length. In more subapical regions GE abundance was about half that near the tip, and this lower level persisted for tens of micrometres. In cells with the hypA1 restrictive phenotype, 


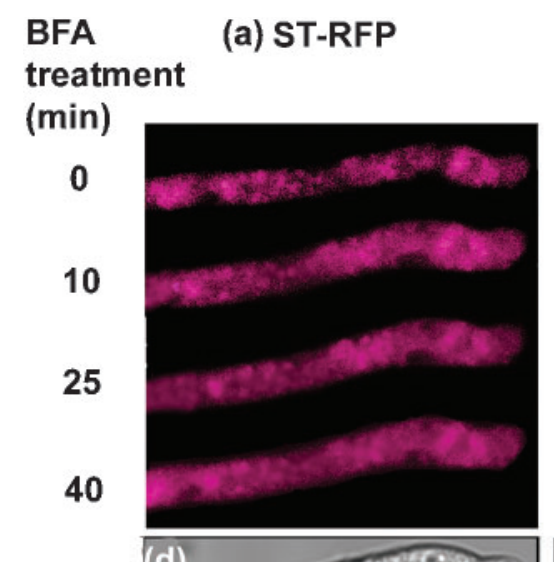

(b) CopA:GFP

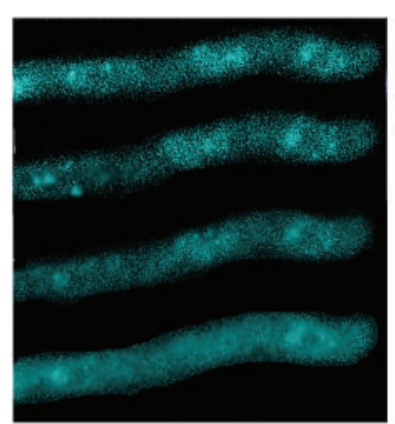

(c) Merge

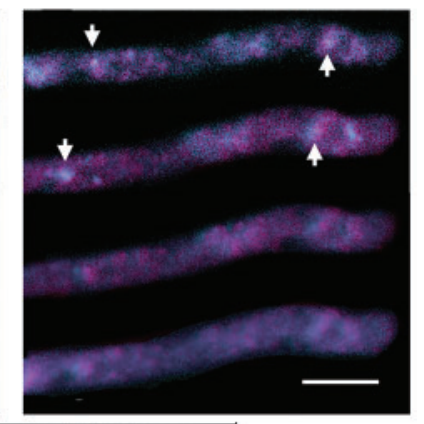

CopA:GFP

Fig. 2. Effect of BFA on fluorescent protein-tagged GE marker distributions in a wild-type-morphology $A$. nidulans hypha. The numbers indicate the time in minutes since the beginning of BFA treatment, which inhibited tip growth. (a) The GE transmembrane marker ST-RFP (magenta) and (b) CopA-GFP (cyan) have predominantly punctate localizations prior to BFA treatment. (c) Merged images of ST-RFP and CopA-GFP. Arrowheads indicate GEs, the appearance of which became more diffuse following BFA treatment. (d) Transmitted light image of the hypha in $(\mathrm{a}-\mathrm{c})$ corresponding to the 40 min BFA treatment. The distribution of both ST-RFP and CopA-GFP became more diffuse within 25 min of treatment. (e) Hypha treated with BFA and imaged for CopA-GFP after $60 \mathrm{~min}$, showing development of putative reticulate labelling patterns (arrows) that were unlike the patterns at 0-10 min. Bar in (c), $5 \mu \mathrm{m}$ for all parts.

the growing tip was tapered and clearly distinguishable from the rounded spore-end of the germling. In hypAl restrictive-phenotype cells, the region of high GE abundance was clearly at the growing tip end.

\section{A. nidulans GEs are mobile, independent of tip growth}

A. nidulans GEs were mobile within the near-apical cytoplasm, showing randomly directed saltatory movements. Some individual GEs could be tracked between frames of time-lapse series of single near-median optical sections, and were found to move independently in all directions (see Supplementary Fig. S1). We defined GE movements as anterograde, retrograde or lateral (Fig. 3a), by comparing their positions between sequential frames. Transmitted light images were used to measure growth rate after the series were collected. GE motion was faster in growing hyphae, and tended to be anterograde (Fig. 3c). Rates were similar in strains with wild-type (AMH1, $\mathrm{AMH} 2$ ) and hypA1 (AAB1) backgrounds grown at $28^{\circ} \mathrm{C}$. GE movements were examined in repolarizing hypA1 cells, which had been grown for $14 \mathrm{~h}$ at $42{ }^{\circ} \mathrm{C}$ (at which temperature they form viable but poorly polarized cells) and then shifted to $28{ }^{\circ} \mathrm{C}$. Under these conditions, new branches visible with transmitted light formed within $1 \mathrm{~h}$ (Fig. 3b). These would later grow into wild-type-morphology hyphae.

In repolarizing hypA1 phenotype cells, anterograde GE movements were about twice as fast (Fig. 3c) and twice as frequent (Fig. 3c) as those in other directions. Rates of anterograde GE movement were most different between poorly (grown at $42{ }^{\circ} \mathrm{C}$ ) and highly polarized (grown at $28{ }^{\circ} \mathrm{C}$ ) cells. There was a clear trend towards an increasing rate of anterograde movement during repolarization (Fig. $3 \mathrm{c})$, but not towards retrograde and lateral movements (Fig. 3b) over the same time-course. Since anterograde GE movements seem intuitively to be the most immediately relevant to tip growth, the remaining data will focus on these.

Fig. 3(d) compares tip growth rates and anterograde GE movement rates in hypA1 cells growing at permissive and restrictive temperatures, and during cell repolarization, a more detailed analysis of the anterograde data shown in Fig. 3(c). The average anterograde GE movement rate was always at least tenfold faster than growth rate. In other words, there was a net anterograde movement of GEs (Fig. $3 \mathrm{~d}$ ), consistent with delivery of growth-related materials to the cell tip.

\section{Cytoskeletal involvement in A. nidulans GE movements}

GE movement will depend on the actin or microtubule (MT) cytoskeletons, or on both. We treated A. nidulans hyphae with actin-depolymerizing (latrunculin), or MTdepolymerizing (benomyl) or -stabilizing (taxol) drugs to study the relative effects on GE movements. The final drug concentrations were prepared from concentrated stock solutions in solvent, so we compared drug effects with 
(a)

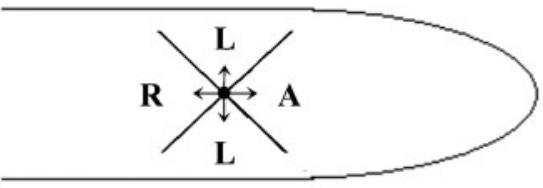

(b)

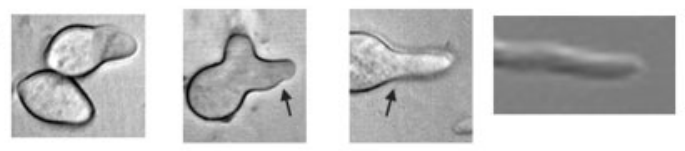

(c)

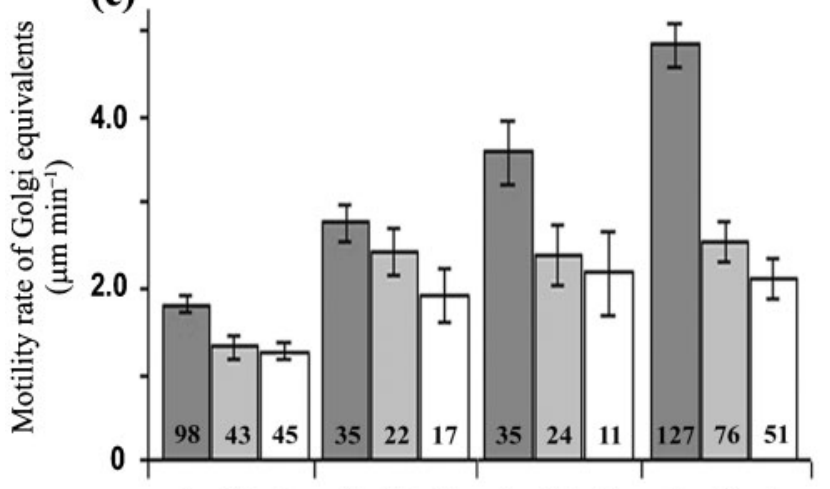

$\begin{array}{llllllllllllll}\mathbf{A} & \mathbf{R} & \mathbf{L} & \mathbf{A} & \mathbf{R} & \mathbf{L} & \mathbf{A} & \mathbf{R} & \mathbf{L} & \mathbf{A} & \mathbf{R} & \mathbf{L}\end{array}$

$42{ }^{\circ} \mathrm{C}$

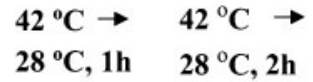

(d)

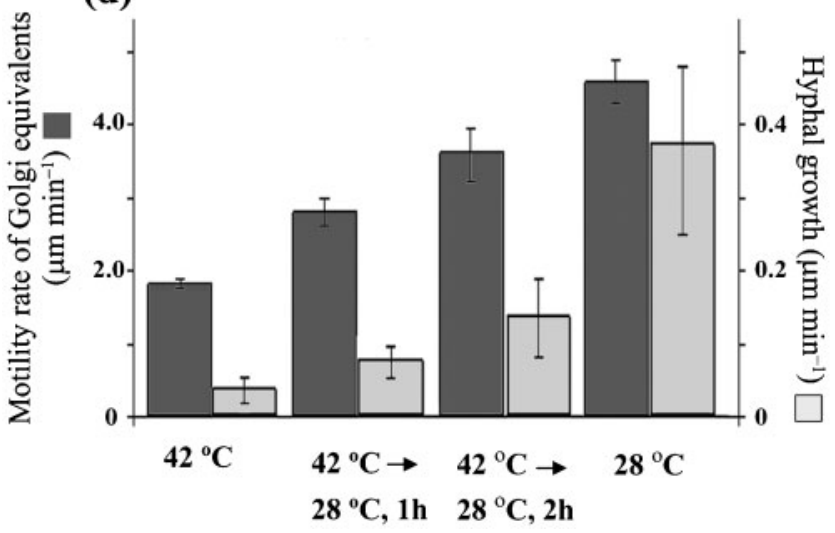

those for solvent-treated control cells. Preliminary studies comparing GE movement and hyphal growth rates for strain $\mathrm{AAB1}$ growing on $\mathrm{CM}$-ethanol, $\mathrm{CM}$-glycerol and nutrient broth-threonine showed no significant difference in GE movement or hyphal growth rate between different growth media (Fig. 4a; data not shown).

Hyphae treated with $5 \mathrm{mg}$ latrunculin $\mathrm{ml}^{-1}$ had a minimal rate of tip growth (Fig. 4b), consistent with the importance of an intact actin cytoskeleton for many cellular processes. Anterograde GE movement in latrunculin-treated cells was significantly reduced compared with controls, but was not abolished (Fig. 4b). Hyphae treated with $1 \mu \mathrm{g}$ benomyl $\mathrm{ml}^{-1}$, which we had shown previously depolymerizes
Fig. 3. Direction and speed of motion of $A$. nidulans GEs, localized with CopA-GFP, in $42{ }^{\circ} \mathrm{C}$-grown hypA1 restrictivephenotype cells that were allowed to repolarize following a shift to $28^{\circ} \mathrm{C}$. (a) Direction of GE movement with respect to the hyphal tip: anterograde $(A)$, retrograde $(R)$ or lateral $(L)$, as described in Methods. (b) Photomicrographs showing representative morphologies of hypA1 and repolarizing phenotypes. The arrows indicate new growth following a shift from 42 to $28^{\circ} \mathrm{C}$. GE movement is shown in repolarizing $A$. nidulans hyp $A 1$ cells, which produce wildtype branches (arrows indicate the growth transition associated with the shift from 42 to $28{ }^{\circ} \mathrm{C}$ ). (c) GE motility in hypA1 restrictive and repolarizing $A$. nidulans AAB1 cells. Spores were grown for $14 \mathrm{~h}$ at $42{ }^{\circ} \mathrm{C}$. For the repolarizing phenotypes, AAB1 spores were grown at $42{ }^{\circ} \mathrm{C}$ for $14 \mathrm{~h}$, then shifted to $28^{\circ} \mathrm{C}$ for 1 or $2 \mathrm{~h}$. For the permissive phenotype, AAB1 spores were grown at $28{ }^{\circ} \mathrm{C}$. Bars indicate rate of $\mathrm{GE}$ movement \pm SEM for each direction, with the numbers indicating the total number of GEs analysed from five cells. GE movement was predominantly anterograde, and was significantly faster than movement in other directions. (d) Anterograde GE movement (dark-grey bars) compared with tip growth rate (light-grey bars) for the cells shown in (c) was always at least 10 -fold higher than the growth rate; note that the $y$-axis scales are 10-fold different. Both GE movement and growth rates increased significantly during cell repolarization. Error bars show SEM.

all cytoplasmic MTs within 2 min but does not prevent hyphal tip growth within $1 \mathrm{~h}$ (Hubbard \& Kaminskyj, 2007), had reduced tip growth rates, but significantly increased anterograde GE movement (Fig. 4b). Hyphae treated with $50 \mu \mathrm{M}$ taxol, which we had shown previously significantly increases cytoplasmic MT abundance (Hubbard \& Kaminskyj, 2007), significantly increased anterograde GE movement rate, but without increasing hyphal growth rate (Fig. 4c). All these drugs caused hyphal morphological abnormalities after several hours; however, we collected growth and GE data only within the first $1 \mathrm{~h}$ (benomyl) or $2 \mathrm{~h}$ (latrunculin and taxol) of treatment.

\section{DISCUSSION}

This is believed to be the first study of GE localization, distribution and behaviour in living A. nidulans hyphae. CopA-GFP and ST-RFP localizations were typically round in optical section, but occasionally appeared to be oval or horseshoe-shaped (see figure 3-2 in Hubbard, 2007). These were consistent with Aspergillus GEs imaged with TEM, which have single pleiomorphic cisternae (Beckett et al., 1974; Kaminskyj \& Boire, 2004; Kurtz et al., 1994), similar to those of Pisolithus (Xu et al., 2004) and Schizophyllum (Rupeš et al., 1995). Fluorescent structures are luminous, which enhances detection and can increase apparent size (Hubbard \& Kaminskyj, 2007). The apparent size of A. nidulans GEs is also consistent with results from plants, which have numerous, motile Golgi bodies (Boevink et al., 1998).

The CaMV promoter has been shown to induce gene expression in plants (Jefferson et al., 1987; Odell \& Nagy, 


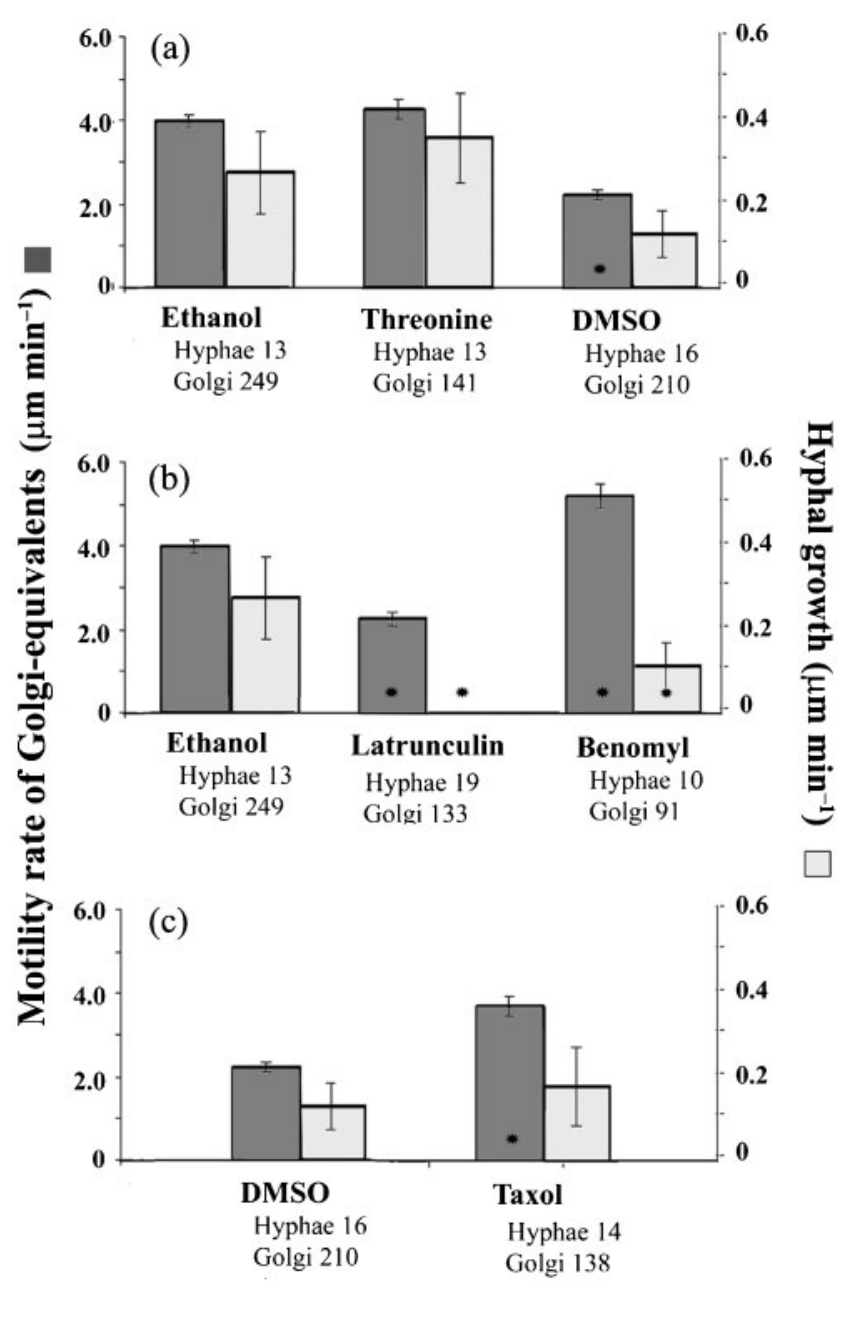

Fig. 4. Effect of solvents and cytoskeleton-inhibitory drugs on anterograde GE movement (dark-grey bars) and hyphal growth rates (light-grey bars) in wild-type-phenotype $A$. nidulans hyphae of strain AAB1 grown at $28{ }^{\circ} \mathrm{C}$. Error bars show SEM. The rate of anterograde GE movement was always at least 10-fold higher than the growth rate in the same cells; note that the $y$-axis scales are 10-fold different. Retrograde and lateral movements were not significantly affected. Asterisks indicate a significant change compared with the respective control $(P<0.05$, ANOVA). (a) Effect of the $A / c A$ inducers $1.0 \%$ ethanol and $0.5 \%$ threonine, and of $0.25 \%$ DMSO in $1.0 \%$ ethanol. The rates of GE movement and growth were similar for ethanol and threonine, but DMSO caused a significant reduction in GE movement but not growth rate. (b) Effect of $5 \mu \mathrm{g}$ latrunculin $\mathrm{B} \mathrm{ml}{ }^{-1}$ and $1 \mu \mathrm{g}$ benomyl $\mathrm{ml}^{-1}$, which target actin and MTs, respectively. Latrunculin treatment significantly reduced both GE movement and growth rate, whereas benomyl treatment increased GE motility while reducing growth rate. (c) Effect of $50 \mu \mathrm{M}$ taxol in $0.25 \%$ DMSO. Compared with the control treatment, taxol significantly increased GE anterograde movement rate without increasing hyphal growth rate.

1985; Wee et al., 1998) and in fungal species, including $S$. cerevisiae (Hirt et al., 1990), Uromyces appendiculatus ( $\mathrm{Li}$ et al., 1993), Ganoderma lucidum and Pleurotus citrinopileatus
(Sun et al., 2002), and Pleurotus ostreatus (Xu et al., 2004). This is, to our knowledge, the first demonstration of its efficacy in $A$. nidulans.

The transmembrane domain of ST has been shown to be associated with Golgi bodies in animal (Munro, 1991), plant (Wee et al., 1998) and fungal (Schwientek et al., 1995) systems. This is, to our knowledge, the first demonstration of its localization in A. nidulans, in which the ST-RFP fluorescence labelling pattern was clearly consistent with that of CopA-GFP labelling. Thus, STRFP is likely to be useful for tracking $A$. nidulans GEs in order to examine their behaviour in living cells without the pharmacological effects noted previously with fluorescent BFA labelling in Pisolithus (Cole et al., 2000), Pleurotus (Xu et al., 2004) and Schizophyllum (Rupeš et al., 1995).

The effect of BFA on CopA-GFP fluorescence distribution in living hyphae was consistent with that shown in Breakspear et al. (2007), and with changes seen with STRFP. BFA blocks the function of a Sec7 guanine nucleotide exchange factor (Donaldson et al., 1992), which is required early in COPI coat formation (Helms \& Rothman, 1992). Within $25 \mathrm{~min}$ of applying BFA, both the ST-RFP and CopA-GFP patterns became less distinct, as expected, since BFA dissociates COPI from vesicles and later affects GE morphology by perturbing vesicle trafficking (Jackson \& Casanova, 2000). Khalaj et al. (2001) showed that $100 \mu \mathrm{g}$ BFA ml $\mathrm{m}^{-1}$ induces perinuclear endomembrane redistributions in Aspergillus niger after $2 \mathrm{~h}$. Our milder and shorter BFA treatments had less extreme effects, but were consistent with more dramatic redistributions over longer timescales.

\section{Distribution and motility of $\boldsymbol{A}$. nidulans GEs}

Most cellular constituents (turgor being a notable exception) show a tip-high gradient of abundance in growing hyphae, although the length and steepness of the individual gradients vary (Heath \& Kaminskyj, 1989). Organelle distributions can be altered by cytoplasmic contractions during chemical fixation (Kaminskyj et al., 1992), so we examined GE distributions in living $A$. nidulans hyphae. In near-median optical sections, fluorescence-localized GE populations were similar to those shown elsewhere with freeze-substitution TEM (Kurtz et al., 1994). In addition, Breakspear et al. (2007) showed a similar GE abundance pattern in fixed hyphae. A. nidulans GEs are appropriately distributed to contribute to apical growth, being relatively more abundant in the near-apical cytoplasm.

In growing hyphae, the near-apical cytoplasm appears to move as a unit (sometimes called bulk flow) with respect to the lateral hyphal walls, using actin-dependent processes (Heath, 1990, 1995; Kaminskyj \& Heath, 1996). Within the cytoplasm, independent nuclear (Morris et al., 1995; Suelmann \& Fischer, 2000b) and mitochondrial (Suelmann \& Fischer, 2000a) movements are generated by cytoskeletondependent processes. When cytoskeletal function was 
perturbed by benomyl, latrunculin or taxol, there was a trend for GEs to become relatively more concentrated near hyphal tips (data not shown), also seen in Breakspear et al. (2007) following nocodazole treatment. This is consistent with a tendency for cytoplasm to contract toward the apex following cytoplasm perturbation (Kaminskyj et al., 1992; Kaminskyj \& Heath, 1996).

The GE distribution pattern in Aspergillus correlates with that of cytoplasmic MTs, which is circumstantial evidence suggesting that longitudinal (but perhaps not lateral) GE motility could use MT-dependent motors. However, unexpectedly, both cytoplasmic MT depolymerization with benomyl and MT polymerization with taxol increased anterograde GE motility. Breakspear et al. (2007) showed that the $n u d A 1^{\text {ts }}$ cytoplasmic dynein heavy chain mutation does not affect GE positioning at restrictive temperature, despite substantial effects on nuclear positioning, suggesting that nuclear and GE motility are differently regulated.

In contrast to their lack of dependence on MTs, Aspergillus GE movement and tip growth are exquisitely dependent on the actin cytoskeleton. Actin-dependent motility has also been shown for plant Golgi bodies (Boevink et al., 1998). A. nidulans hyphae treated for more than $2 \mathrm{~h}$ with any of these cytoskeleton-targeting drugs began to develop morphological abnormalities (Hubbard, 2007; Hubbard \& Kaminskyj, 2007), suggesting that wall deposition and cell extension can continue despite sublethal cytoskeletal impairment, albeit with aberrant apical targeting. Clearly, the mechanisms underlying GE motility will prove to be complex.

\section{Anterograde transport of tip growth-related materials}

Given the size of an A. nidulans wall vesicle $(\sim 50 \mathrm{~nm}$ diameter) and the area of cell membrane required for a growth rate of about $0.5 \mu \mathrm{m} \mathrm{min}^{-1}$ (Hubbard \& Kaminskyj, 2007) of a hypha about $3 \mu \mathrm{m}$ in diameter (Kaminskyj \& Hamer, 1998), at least 600 vesicles per minute must fuse at the hyphal tip. If long-distance anterograde transport of growthrelated materials in Aspergillus was in vesicles, then vesicle abundance should be relatively consistent in near-apical cytoplasm. However, TEM shows that Aspergillus hyphae have a substantially larger vesicle population in the apical 2$3 \mu \mathrm{m}$ compared with even a few micrometres slightly further back (see figure 4a in Kurtz et al., 1994). Thus, long-distance wall material transport in vesicles is not consistent with vesicle distribution patterns in Aspergillus hyphae. This is unlike Saprolegnia ferax, where individual wall vesicles produced by Golgi bodies located in the central cytoplasm appear to migrate to and then be transported along the cell periphery (Heath \& Kaminskyj, 1989). Fungal and oomycete hyphae have similar shapes, and the organisms have comparable lifestyles, but they are phylogenetically distant.

There are about 15 cytoplasmic MTs or MT bundles in the apical $20 \mu \mathrm{m}$ of an A. nidulans hypha (Hubbard \&
Kaminskyj, 2007). If A. nidulans growth materials were transported in vesicles, along MTs, vesicle distributions visualized with electron tomography should be spatially correlated with cytoplasmic MTs. However, figure 2 in Hohmann-Marriott et al. (2006) does not provide support for this relationship. In addition, the average separation between vesicles and MTs is about $50 \mathrm{~nm}$, twice the length expected for cytoplasmic dynein (R. Roberson, personal communication). Vesicle distribution in Aspergillus hyphae does not appear to be spatially correlated with cytoplasmic MTs, arguing against a direct role for MTs in vesicle motility.

It has generally been assumed that wall-forming materials in filamentous fungi are transported to hyphal tips in vesicles, by means of cytoplasmic MT-dependent motors (Bartnicki-Garcia, 2002; Breakspear et al., 2007; Fischer \& Veith, 2007). However, direct evidence to support this model is sparse, or argues to the contrary (HohmannMarriott et al., 2006). Results described in Hubbard \& Kaminskyj (2007) suggest that tip growth rates in $A$. nidulans are not quantitatively related to cytoplasmic MT number. Vesicle transport could also be mediated by actin, which has multiple roles in tip growth (Bartnicki-Garcia, 2002; Harris et al., 1994; Heath, 1990, 1995; Kaminskyj \& Heath, 1996). The mechanisms underlying organelle movement in A. nidulans have not been explored, apart from those of MTs in nuclear (Morris et al., 1995; Suelmann \& Fischer, 2000b) and actin in mitochondrial motility (Suelmann \& Fischer, 2000a). Our data showing that A. nidulans GEs have predominant anterograde motility at rates far exceeding the rate of tip growth in the same cells suggest that growth-related materials might be transported to the tip packaged in GEs.

In contrast to a model proposing long-distance transport of wall vesicles in A. nidulans, the pattern of tip-localized vesicle abundance in Aspergillus hyphal tips is complementary to that of Aspergillus GEs (Kurtz et al., 1994; Breakspear et al., 2007; this paper). This then is consistent with the notion that GEs offload their contents in the vicinity of the Spitzenkörper, presumably as vesicles, prior to changes that permit them to fuse at the hyphal apex. In A. nidulans hyphae examined following diverse treatments, anterograde GE movement was consistently at least 10 -fold faster than tip growth rates. This suggests that GEs may be delivering wall-building materials to near-apical regions of the hypha, potentially supplementing (or supplanting?) bulk delivery by individual vesicles.

\section{A model for the role of GE movement in A. nidulans tip growth}

The model shown in Fig. 5 concerns the role of GE motility in A. nidulans hyphal growth. As discussed earlier, fungal GEs have a function analogous to that of Golgi bodies in animals and plants. Endoplasmic reticulum (ER) to GE transport in fungi (Fig. 5a) might occur at specialized sites, as has been proposed for yeasts (Bevis et al., 2002; 


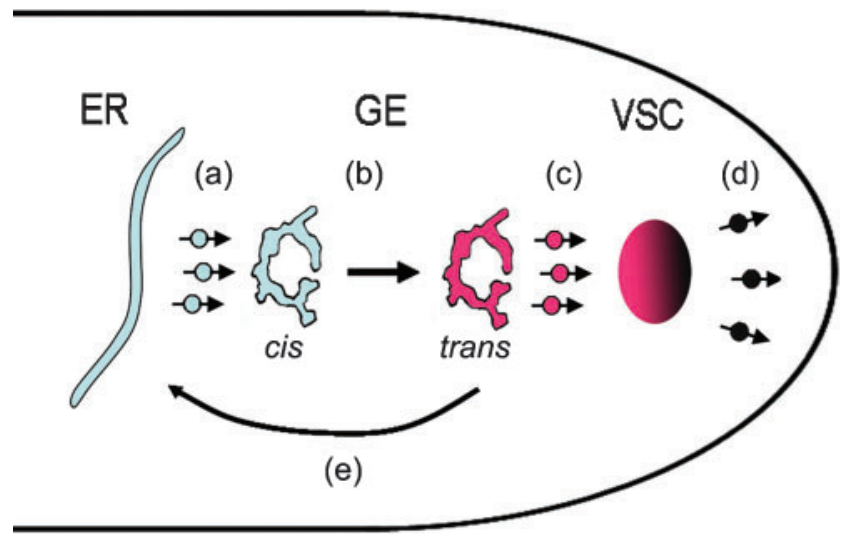

Fig. 5. Model describing how anterograde GE movement might contribute to hyphal tip growth. Aspergillus GEs are single pleiomorphic cisternae; this cartoon was adapted from a freezesubstitution transmission electron micrograph (see figure $4 \mathrm{a}$ in Kurtz et al., 1994). (a) Endoplasmic reticulum (ER) to GE transport. GEs are transported toward the hyphal tip (b) at rates roughly 10fold higher than that of tip growth. Maturation of cis-Golgi to transGolgi (indicated by colour change) may occur during anterograde transport. GEs do not accumulate at the hyphal tip because they unload their cargo (c) near the Spitzenkörper (vesicular supply centre; VSC). Changes in vesicle membranes associated with the VSC (indicated by colour gradient; individual vesicles are not shown) permit them to fuse with the cell membrane for secretion at the hyphal tip (d). Currently it is unclear whether the Golgi are disassembled or recycled following uploading. If GEs that have unloaded their cargo are recycled to subapical regions to collect more cargo, this could comprise a portion of the lateral or retrograde GE motility (e). Stages (a) and (c) and transit from the VSC to the cell membrane are depicted as being predominantly mediated by vesicle traffic, although direct ER-GE transport by transfer ER has been postulated for plants and certain yeasts.

Mogelsvang et al., 2003) and plants (daSilva et al., 2004; Matheson et al., 2006). Both the ER (Fernández-Ábalos et al., 1998; Maruyama \& Kitamoto, 2007) and GEs (this paper; Breakspear et al., 2007) are widely distributed in the apical cytoplasm of $A$. nidulans hyphae. Anterograde GE transport may be accompanied by maturation from cis-like to trans-like GE elements (Fig. 5b), a functional analogy to cisternal maturation in Golgi bodies, consistent with changes recently documented in yeast (Losev et al., 2006; Matsuura-Tokita et al., 2006). Despite predominantly anterograde GE movement, GEs do not accumulate at the tips of $A$. nidulans hyphae. Indeed, GEs were notably less abundant within $5 \mu \mathrm{m}$ of the tip, suggesting that they offload their cargo (Fig. 5c), presumably as vesicles destined for the Spitzenkörper (vesicle supply centre; VSC). VSC- and exocytosis-related events (Fig. 5d) are shown for completeness, but are not directly addressed by this model. Following unloading, GEs might be transported subapically to acquire more growth-related materials (Fig. $5 \mathrm{e})$, which could account for at least some retrograde and lateral movements. Studies to investigate the events involved in the loading, transport and unloading of GEs are under way.

\section{ACKNOWLEDGEMENTS}

This research was supported by a Natural Sciences and Engineering Research Council (NSERC) grant to S.G.W.K., which is gratefully acknowledged. M. A. H. was supported by a University of Saskatchewan Dean's Graduate Scholarship and an NSERC Canada Graduate Scholarship (Masters). The Canada Foundation for Innovation is acknowledged for purchase of the Zeiss META510 confocal microscope. The Brandizzi (formerly University of Saskatchewan) and Hawes (Oxford Brookes University) groups supplied the ST-RFP plasmid. The Assinder group (University of Wales, Bangor, UK) provided the CopAGFP, hypA1 strain. We thank Xiaohui Bao for translating Xu et al. (2004), and anonymous reviewers for useful comments.

\section{REFERENCES}

Akao, T., Yamaguchi, M., Yahara, A., Yoshiuchi, K., Fujita, H., Yamada, O., Akita, O., Ohmachi, T., Asada, Y. \& Yoshida, T. (2006). Cloning and expression of 1,2- $\alpha$-mannosidase gene (fmanIB) from filamentous fungus Aspergillus oryzae: in vivo visualization of the FmanIBp-GFP fusion protein. Biosci Biotechnol Biochem 70, 471-479.

Bartnicki-Garcia, S. (2002). Hyphal tip growth: outstanding questions. In Molecular Biology of Fungal Development, pp. 29-58. Edited by H. D. Osiewacz. Boca Raton, FL: CRC Press.

Beckett, A., Heath, I. \& McLaughlin, D. J. (1974). An Atlas of Fungal Ultrastructure. London: Longman.

Bentivoglio, M. \& Mazzarello, P. (1998). The pathway to the cell and its organelles: one hundred years of the Golgi apparatus. Endeavour 22, 101-105.

Bevis, B. J., Hammond, A. T., Reinke, C. A. \& Glick, B. S. (2002). De novo formation of transitional ER sites and Golgi structures in Pichia pastoris. Nat Cell Biol 4, 750-756.

Boevink, P., Oparka, K., Cruz, S. S., Martin, B., Betteridge, A. \& Hawes, C. (1998). Stacks on tracks: the plant Golgi apparatus traffics on an actin. Plant J 15, 441-447.

Breakspear, A., Langford, K., Momany, M. \& Assinder, S. S. (2007). CopA:GFP localizes to putative Golgi equivalents in Aspergillus nidulans. FEMS Microbiol Lett 277, 90-97.

Cole, L., Davies, D., Hyde, G. J. \& Ashford, A. E. (2000). Brefeldin A affects growth, endoplasmic reticulum, Golgi bodies, tubular vacuole system, and secretory pathway in Pisolithus tinctorius. Fungal Genet Biol 29, 95-106.

daSilva, L. L. P., Snapp, E. L., Denecke, J., Lippincott-Schwartz, J., Hawes, C. \& Brandizzi, F. (2004). Endoplasmic reticulum export sites and Golgi bodies behave as single mobile secretory units in plant cells. Plant Cell 16, 1753-1771.

Donaldson, J. G., Finazzi, D. \& Klausner, R. D. (1992). Brefeldin A inhibits Golgi-membrane catalyzed exchange of guanine nucleotide onto ARF protein. Nature 360, 350-352.

Farquhar, M. G. \& Palade, G. E. (1981). The Golgi apparatus (complex) - (1954-1981) from artifact to center stage. J Cell Biol 91, 77s-103s.

Farquhar, M. G. \& Palade, G. E. (1998). The Golgi apparatus: 100 years of progress and controversy. Trends Cell Biol 8, 2-10.

Felenbok, B. (1991). The ethanol utilization regulon of Aspergillus nidulans: the AlcA-AlcR system as a tool for the expression of recombinant proteins. J Biotechnol 17, 11-18. 
Fernández-Ábalos, J. M., Fox, F., Pitt, C., Wells, B. \& Doonan, J. H. (1998). Plant-adapted green fluorescent protein is a versatile vital reporter for gene expression, protein localization and mitosis in the filamentous fungus, Aspergillus nidulans. Mol Microbiol 27, 121-130.

Fischer, R. \& Veith, D. (2007). The role of microtubules and motors for polarized growth of filamentous fungi. In Exploitation of Fungi: Symposium of the British Mycological Society held at the University of Manchester September 2005 (British Mycological Society Symposia no. 26), pp. 95-116. Edited by G. D. Robson, P. Van West \& G. M. Gadd. Cambridge: Cambridge University Press.

Harris, S. D., Morrell, J. L. \& Hamer, J. E. (1994). Identification and characterization of Aspergillus nidulans mutants defective in cytokinesis. Genetics 136, 517-532.

Hawes, C. \& Satiat-Jeunemaitre, B. (2005). The plant Golgi apparatus - going with the flow. Biochim Biophys Acta 1744, 93-107.

Heath, I. B. (1990). The roles of actin in tip growth of fungi. Int Rev Cytol 123, 95-127.

Heath, I. B. (1995). The cytoskeleton. In The Growing Fungus, 1st edn, pp. 99-134. Edited by N. A. R. Gow \& G. M. Gadd. London: Chapman and Hall.

Heath, I. B. \& Kaminskyj, S. G. W. (1989). The organization of tipgrowth-related organelles and microtubules revealed by quantitative analysis of freeze-substituted oomycete hyphae. J Cell Sci 93, 41-52.

Helms, J. B. \& Rothman, J. E. (1992). Inhibition by brefeldin A of a Golgi membrane enzyme that catalyses exchange of guanine nucleotide bound to ARF. Nature 360, 352-354.

Hirt, H., Kogl, M. \& Murbacher, T. (1990). Evolutionary conservation of transcriptional machinery between yeast and plants as shown by the efficient expression from the CaMV $35 \mathrm{~S}$ promoter and $35 \mathrm{~S}$ terminator. Curr Genet 17, 473-479.

Hohmann-Marriott, M. F., Uchida, M., van de Meene, A. M. L., Garret, M., Hjelm, B. E., Kokoori, S. \& Roberson, R. W. (2006). Application of electron tomography to fungal ultrastructure studies. New Phytol 172, 208-220.

Hubbard, M. \& Kaminskyj, S. G. W. (2007). Growth rate of Aspergillus nidulans hyphae is independent of a prominent array of microtubules. Mycol Prog 6, 179-189.

Hubbard, M. (2007). In vivo study of the role of the cytoskeleton and fungal Golgi in hyphal tip growth of Aspergillus nidulans. MSc thesis, University of Saskatchewan. Available at library2.usask.ca/theses/ available/etd-05042007-171757

Jackson, C. L. \& Casanova, J. E. (2000). Turning on ARF: the Sec7 family of guanine-nucleotide-exchange factors. Trends Cell Biol 10, 60-67.

Jefferson, R. A., Kavanagh, T. A. \& Bevan, M. W. (1987). GUS fusions: $\beta$-glucuronidase as a sensitive and versatile gene fusion marker in higher plants. EMBO J 6, 3901-3908.

Kaminskyj, S. G. W. (2001). Fundamentals of growth, storage, genetics and microscopy of Aspergillus nidulans. Fungal Genet Newsl 48, 25-31.

Kaminskyj, S. G. W. \& Boire, M. R. (2004). Ultrastructure of the Aspergillus nidulans hypA1 restrictive phenotype shows defects in endomembrane arrays and polarized wall deposition. Can J Bot 82, 807-814.

Kaminskyj, S. G. W. \& Hamer, J. E. (1998). hyp loci control cell pattern formation in the vegetative mycelium of Aspergillus nidulans. Genetics 148, 669-680.

Kaminskyj, S. G. W. \& Heath, I. B. (1996). Studies on Saprolegnia ferax suggest the general importance of the cytoplasm in determining hyphal morphology. Mycologia 88, 20-37.
Kaminskyj, S. G. W., Jackson, S. L. \& Heath, I. B. (1992). Fixation induces differential polarized translocations of organelles in hyphae of Saprolegnia ferax. J Microsc 167, 153-168.

Khalaj, V., Brookman, J. L. \& Robson, G. D. (2001). A study of the protein secretory pathway of Aspergillus niger using a glucoamylaseGFP fusion protein. Fungal Genet Biol 32, 55-65.

Kurtz, M. B., Heath, I. B., Marrinan, J., Dreikorn, S., Onishi, J. \& Douglas, C. (1994). Morphological effects of lipopeptides against Aspergillus fumigatus correlate with activities against (1,3)- $\beta$-Dglucanase. Antimicrob Agents Chemother 38, 1480-1489.

Li, A., Altosaar, I., Heath, M. C. \& Horgen, P. A. (1993). Transient expression of the beta-glucuronidase gene delivered into urediniospores of Uromyces appendiculatus by particle bombardment. Can J Plant Pathol 15, 1-6.

Losev, E., Reinke, C. A., Jellen, J., Strongin, D. E., Bevis, B. J. \& Glick, B. S. (2006). Golgi maturation visualized in living yeast. Nature 441, 1002-1006.

Maruyama, J. \& Kitamoto, K. (2007). Differential distribution of the endoplasmic reticulum network in filamentous fungi. FEMS Microbiol Lett 272, 1-7.

Matheson, L. A., Hanton, S. L. \& Brandizzi, F. (2006). Traffic between the plant endoplasmic reticulum and Golgi apparatus: to the Golgi and beyond. Curr Opin Plant Biol 9, 601-609.

Matsuura-Tokita, K., Takeuchi, M., Ichihara, A., Mikuriya, K. \& Nakano, A. (2006). Live imaging of yeast Golgi cisternal maturation. Nature 441, 1007-1010.

McGoldrick, C. A., Gruver, C. \& May, G. S. (1995). myoA of Aspergillus nidulans encodes an essential myosin I required for secretion and polarized growth. J Cell Biol 128, 577-587.

Mogelsvang, S. \& Howell, K. E. (2006). Global approaches to study Golgi function. Curr Opin Cell Biol 18, 438-443.

Mogelsvang, S., Gomez-Ospina, N., Soderholm, J., Glick, B. S. \& Staehelin, L. A. (2003). Tomographic evidence for continuous turnover of Golgi cisternae in Pichia pastoris. Mol Biol Cell 14, 2277-2291.

Morris, N. R., Xiang, X. \& Beckwith, S. M. (1995). Nuclear migration advances in fungi. Trends Cell Biol 5, 278-282.

Munro, S. (1991). Sequences within and adjacent to the transmembrane segment of $\alpha-2,6$-sialyltransferase specify Golgi retention. EMBO J 10, 3577-3588.

Odell, J. T. \& Nagy, F. (1985). Identification of DNA sequences required for activity of the cauliflower mosaic virus $35 \mathrm{~S}$ promoter. Nature 313, 810-812.

Rida, P. C. G., Nishikawa, A., Won, G. Y. \& Dean, N. (2006). Yeast-tohyphal transition triggers formin-dependent Golgi localization to the growing tip in Candida albicans. Mol Biol Cell 17, 4364-4378.

Rupeš, I., Mao, W. Z., Astrom, H. \& Raudaskoski, M. (1995). Effect of nocodazole and brefeldin-A on microtubule cytoskeleton and membrane organization in the homobasidiomycete Schizophyllum commune. Protoplasma 185, 212-221.

Schwientek, T., Lorenz, C. \& Ernst, J. F. (1995). Golgi localization in yeast is mediated by the membrane anchor region of rat liver sialyltransferase. J Biol Chem 270, 5483-5489.

Sharpless, K. E. \& Harris, S. D. (2002). Functional characterization and localization of the Aspergillus nidulans formin SEPA. Mol Biol Cell 13, 469-479.

Shi, X., Sha, Y. \& Kaminskyj, S. (2004). Aspergillus nidulans hypA regulates morphogenesis through the secretion pathway. Fungal Genet Biol 41, 75-88.

Suelmann, R. \& Fischer, R. (2000a). Mitochondrial movement and morphology depend on an intact actin cytoskeleton in Aspergillus nidulans. Cell Motil Cytoskeleton 45, 42-50. 
Suelmann, R. \& Fischer, R. (2000b). Nuclear migration in fungi: different motors at work. Res Microbiol 151, 247-254.

Sun, L., Cai, H., Xu, W., Hu, Y. \& Lin, Z. (2002). CaMV 35 S promoter directs $\beta$-glucuronidase expression in Ganoderma lucidum and Pleurotus citrinopileatus. Mol Biotechnol 20, 239-244.

Wee, E. G.-T., Sherrier, D. J., Prime, T. A. \& Dupree, P. (1998). Targeting of active sialyltransferase to the plant Golgi apparatus. Plant Cell 10, 1759-1768.
Whittaker, S. L., Lunness, P., Milward, K. J., Doonan, J. H. \& Assinder, S. J. (1999). sodVIC is an $\alpha$-COP-related gene which is essential for establishing and maintaining polarized growth in Aspergillus nidulans. Fungal Genet Biol 26, 236-252.

Xu, X., Mang, J., Li, X., Yu, M. \& Ru, B. (2004). Expression of human intestinal trefoil factor (hITF) in transgenic Pleurotus ostreatus and its ELISA assay. Acta Microbiol Sin 44, 609-612.

Edited by: S. D. Harris 